\title{
Facile fabrication of encryption composite materials with trilayer quasi-amorphous heterostructure
}

\author{
Fantao Meng ${ }^{1}$, Malik Muhammad Umair ${ }^{1}$, Shufen Zhang $^{1}$, Yao Meng ${ }^{2}$ and Bingtao Tang ${ }^{1,2 *}$
}

\begin{abstract}
Encryption materials represent a major direction of information security, but it is challenging to realize the ideal security combining the complete hiding of complex coding, stability in transmission, camouflage, and reliable decoding. Herein, we report an innovative strategy for rationally constructing encryption composite materials with high security and portability. Compared with common colloidal photonic watermarks, our encrypted optical devices are composed of thermoplastic polyurethanes (TPU) protective layers, polystyrene (PS) switching layers and a CdS coding layer. The optical shielding properties and phase state transition of PS layers enable high-level encoding and decoding security through thermal activation of light transmittance control. Meanwhile, the CdS layer can be clearly revealed in decoding mode because of the high refractive index. Besides, the confinement protection of integrated TPU films prevents the trilayer quasi-amorphous heterostructure from damage. To establish the practical advantage, portable information carriers can be prepared for camouflage. Accordingly, the reliable encoding and decoding system, and ability to integrate with easy-to-carry products, make our composites a potentially high-security encryption device for anti-fraud technology.
\end{abstract}

Keywords: quasi-amorphous structures, structural color, encryption

\section{INTRODUCTION}

With the continuous development of information technology in the 21 st century, information security has gained significance in many fields, such as the military and business, and in daily life [1,2]. The essence of information security is to protect information resources from all kinds of threats, interferences, and damages. As an important component of information security, information encryption technology refers to the use of mathematical or physical means in protecting informa- tion from illegal access. In recent years, unique functional materials have been developed for information encryption, including organic afterglow materials with distinguishable lifetime codes or reversible solid-state emission features [3-5], responsive photonic watermarks [6-19], rare-earth-doped materials with luminescence modulation [20], phase-change thermochromic materials [21], and plasmonic metamaterials [22-24]. Although these encryption strategies can be promising tools for strict security protection, their integration into real-life applications still faces great challenges due to their inability to meet some aspects of information security simultaneously. First, for security, encoded information can be designed in complex multimode systems with full concealment under normal conditions. Encrypted materials should possess excellent mechanical properties and flexibility to enable the strong and durable protection of information, and can be preferably constructed into portable products. Finally, a reliable decoding strategy that can effectively load and recover coded information without data loss is necessary.

Recently, a bilayer polymer photonic crystal with shape memory effect has been developed for the water-responsive encoding and decoding of information systems [7]. However, the pressure-assisted fabrication of coding regions increases the effective refractive index due to the change of lattice spacing. As a result, encryption patterns are incompletely hidden. We speculate that such strategies are incompletely reliable because data loss can occur through the simple touch of a finger during processing or transmission. Moreover, encryption patterns can be damaged by sweat, which contains inorganic salts (such as $\mathrm{NaCl}$ ) that promote water penetration and influence the accurate decryption of information. Although bilayer heterostructure can be used to encrypt and decrypt information through solvent response [25] and angle

\footnotetext{
${ }^{1}$ State Key Laboratory of Fine Chemicals, Dalian University of Technology, Dalian 116024, China

${ }^{2}$ Eco-chemical Engineering Cooperative Innovation Center of Shandong, Qingdao University of Science and Technology, Qingdao 266042, China

* Corresponding author (email: tangbt@dlut.edu.cn)
} 
change [26], the decoding methods of these strategies are prone to data breach. Therefore, solving the problems of complete hiding, stability in transmission, and decoding security in practical applications remains challenging. Designing a reliable encryption method that affords robust and portable hiding materials with camouflage ability is important for daily-life applications.

Herein, we report a novel and facile strategy for integrating trilayer quasi-amorphous heterostructure into thermoplastic polyurethanes (TPU) films (Fig. 1a). The encrypted optical devices are robust, portable and easily camouflaged. Importantly, the decryption method under high-temperature conditions is imperceptible in daily environment and independent of other variables; thus it is difficult to crack during transmission, avoiding data leakage and providing high-level information security (Fig. 1c). The mechanism of the design is based on the thermoplasticity of organic microspheres and the thermal stability of inorganic microspheres. It utilizes the light transmittance control of quasi-amorphous structures (QASs) through thermal activation to enable the selective presentation of optical scattering. The composite struc- ture is composed of TPU protective layers, polystyrene (PS) switching layers, and a CdS coding layer. The QAS layer of PS is the key component for switching between encoding and decoding. The shielding effect of multiple light scattering [27-33] of the PS layers in encoding mode completely conceals the encrypted layer of CdS, and the composite structure only displays the structural color of the PS layer [34-36] (Fig. 1c). In the high-temperature decoding, the PS layers become transparent after phase state transition [37,38], allowing the penetration of incident light and scattering light. The QAS layer of CdS can be designed with complex encryption patterns, and a decoded structural color can be easily captured because of the high refractive index of CdS microspheres [39,40]. Patterned structures are tightly packed into TPU films after hot pressing at a lower temperature [41], imparting excellent mechanical stability and flexibility (Fig. 1b). The heterostructure can be easily loaded into portable information carriers, such as phone cases, for camouflage (Fig. 1d). The reliable encoding and decoding system, and ability to integrate with easy-to-carry products through gradient heat treatment, make our composites a poten-

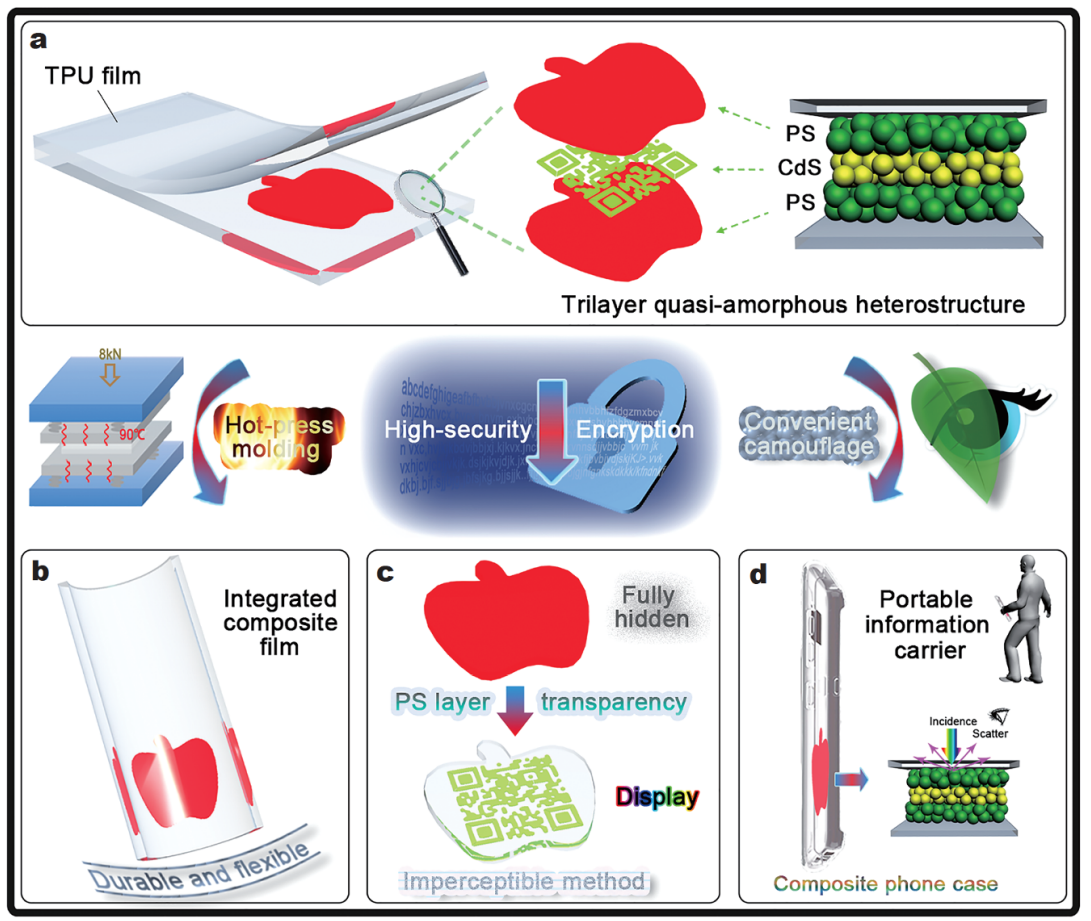

Figure 1 Design and performance of the encrypted optical devices with trilayer quasi-amorphous heterostructure. (a) Schematic diagram of the assembly. (b) The integrated composite structure possesses durability and flexibility due to the confinement of superadhesive TPU films after hotpress molding. (c) Illustration of the encryption and decryption performance based on the optical shielding properties and phase state transition of PS layers. Importantly, the decryption method under high-temperature conditions is imperceptible in daily environment and thus difficult to crack during transmission, avoiding data leakage and providing high-level information security. (d) The heterostructure can be easily loaded into portable information carriers, such as phone cases, to achieve convenient camouflage. 
tially high-security encryption device for anti-fraud technology.

\section{EXPERIMENTAL SECTION}

\section{Preparation of monodispersed PS emulsion}

In a four-port bottle equipped with condensing tube and nitrogen protection device, $62.5 \mathrm{mg}$ sodium dodecyl sulfate (emulsifier) and $135 \mathrm{~mL}$ deionized water were added and then stirred at $300 \mathrm{r} \mathrm{min}^{-1}$ in a water bath of $85^{\circ} \mathrm{C}$ for $15 \mathrm{~min}$. After addition of $15.0 \mathrm{~g}$ styrene monomer, the solution was stirred for another $10 \mathrm{~min}$. Finally, $0.15 \mathrm{~g}$ potassium persulfate was added as the initiator, and the reaction was stirred for $6 \mathrm{~h}$ to obtain white PS emulsion.

\section{Preparation of CdS microspheres}

In a $250-\mathrm{mL}$ beaker, $3.08 \mathrm{~g} \mathrm{Cd}\left(\mathrm{NO}_{3}\right)_{2} \cdot 4 \mathrm{H}_{2} \mathrm{O}, 0.76 \mathrm{~g}$ thiourea, $5 \mathrm{~g}$ polyvinylpyrrolidone and $150 \mathrm{~mL}$ diethylene glycol were added, and stirred at $80^{\circ} \mathrm{C}$ until completely dissolved. The solution was then transferred to a $250-\mathrm{mL}$ three-port bottle and stirred in an oil bath of $160^{\circ} \mathrm{C}$ for $4 \mathrm{~h}$. Finally, the original dispersion was centrifuged and washed three times with water to obtain pure CdS microspheres.

\section{Fabrication of trilayer quasi-amorphous heterostructure} by spraying

The concentration of PS emulsion was adjusted to
$10 \mathrm{wt} \%$, and a certain amount of carbon black (CB) (the mass ratio of $\mathrm{CB} / \mathrm{PS}=0.5 \mathrm{wt} \%$ ) was added to mix evenly by $0.5-\mathrm{h}$ ultrasonic treatment. The concentration of CdS emulsion was also adjusted to $10 \mathrm{wt} \%$. For layer-by-layer construction of the quasi-amorphous heterostructure by spraying, the emulsion was transferred into an airbrush with a $0.2-\mathrm{mm}$ nozzle, and then sprayed onto the TPU film (pre-heated to $50^{\circ} \mathrm{C}$ ) through a patterned mask under $50 \mathrm{kPa}$. The other two layers followed the same procedure. The homogeneous QAS coatings were formed by adjusting the distance and pressure of the airbrush and preheating the substrate.

\section{RESULTS AND DISCUSSION}

Fabrication of portable optical composite devices

A schematic of the fabrication of flexible encryption composites with trilayer QAS patterns is shown in Fig. 2. The top and bottom layers show apple patterns prepared by spraying the mixed emulsion of PS microspheres and $\mathrm{CB}$, and the middle layer with the coded pattern was prepared through the spraying of CdS microspheres (Fig. 2a-f). The sandwich structure containing QAS patterns was formed into an integrated film by hot pressing at $90^{\circ} \mathrm{C}$ and $8 \mathrm{kN}$ (Fig. $2 \mathrm{~g}-\mathrm{j}$ ). The facile spraying method is conducive for designing multiple patterns with complex encrypted information and achieving high privacy. In the composites, two PS layers make the middle coded layer of CdS completely invisible. The wrapped

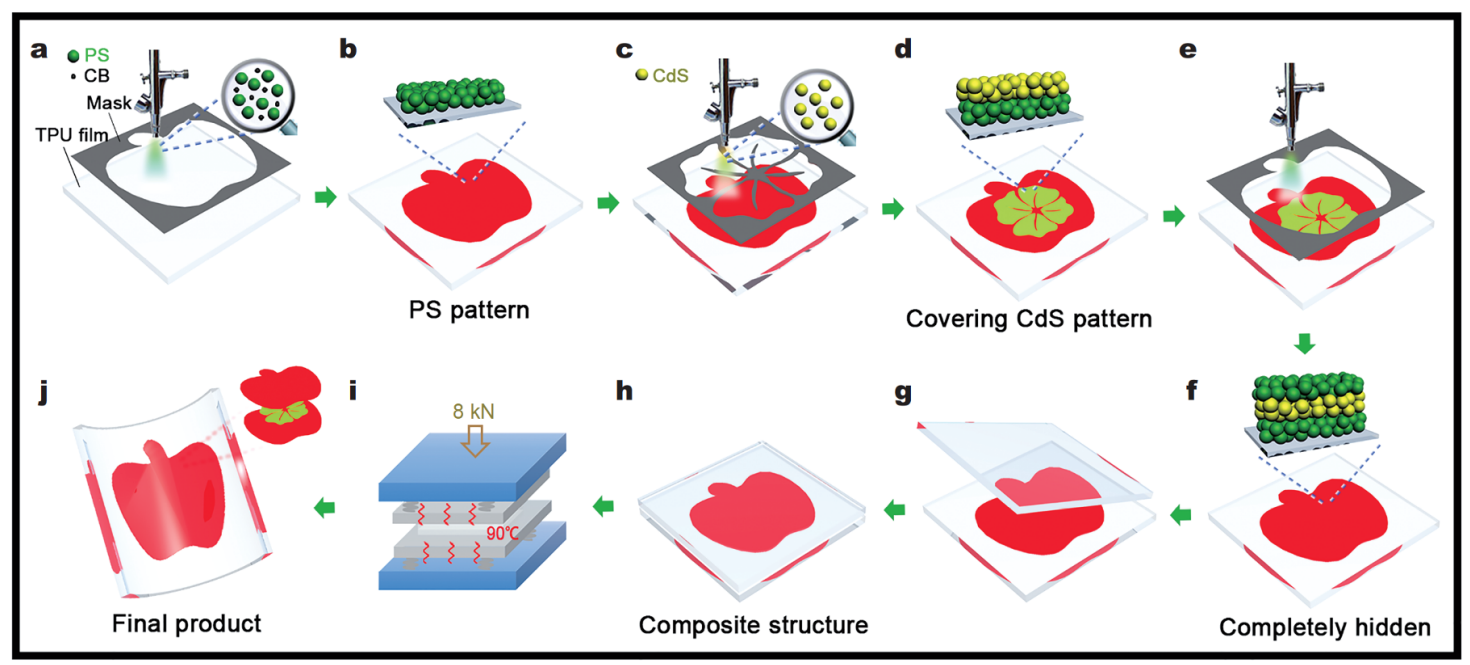

Figure 2 Schematic to fabricate the robust optical composite devices with trilayer QAS patterns. (a) The bottom pattern on the TPU film was formed by spraying the mixed emulsion of PS microspheres and CB through a mask. (b) The bottom PS pattern on the TPU film. (c) The encoded pattern on the PS layer was formed by spraying the CdS emulsion through another mask. (d) Two layers of patterns on the TPU film. (e) The upper PS pattern was constructed in the same way as (a). (f) Trilayer patterned quasi-amorphous heterostructure on the TPU film. (g) Covering by another TPU film. (h) Composite structure to be processed. (i) Hot-press molding of the composite structure. (j) The molded encryption composites. 
TPU films provide the interfacial confinement of QAS patterns induced by thermal superadhesion [41]. Differential scanning calorimetry (DSC) analysis (Fig. S1) revealed that the glass transition temperature $\left(T_{\mathrm{g}}\right)$ of TPU was approximately $-36^{\circ} \mathrm{C}$. Therefore, during gradient heat treatment, TPU films can be hot-pressed at a low temperature (such as $90^{\circ} \mathrm{C}$ ) for the fabrication of portable heat molding materials. Consequently, the morphology of PS microspheres remained intact and the structural color was maintained in the final robust product.

\section{Encryption and decryption mechanism towards high security}

For real information concealment, the encryption layer must be completely covered by screening layers, and no color contrast exists between the encoded information pattern and uncoded areas. The quasi-amorphous arrays composed of microspheres have isotropic pseudo photonic bandgap, the position and width of which hardly change with the angle of incident light. In the pseudo photonic bandgap, the incident light is coherently scattered to produce angle-independent structural color, but the light transmits through the classical diffusion process outside the bandgap $[42,43]$. Therefore, there are multiple incoherent light scattering in the quasi-amorphous arrays. In the as-prepared composite film, the multiple scattering effect of PS layers is expected to form optical shielding [27-33] and completely hide the structural colors of the intermediate layer. Given that the multiple scattering intensities of QASs increase with increasing thickness, the thicknesses of the upper and bottom PS layers play a crucial role in transmittance. The intensity of multiple light scattering is also related to the diameter of the microspheres. The larger the size of PS microspheres is, the stronger the intensity becomes. Taking PS microspheres with average size of $268.6 \mathrm{~nm}$ (Fig. S2) as examples, we prepared several composite structures of PS layers with different thicknesses to determine the shielding degree and the appropriate thickness. The corresponding transmittance spectra of the middle PS layers are presented in Fig. 3a. The thicknesses of PS layers were controlled by adjusting the number of spraying cycles. Using mixed PS emulsion (10 wt \%, the mass ratio of $\mathrm{CB} /$ PS $=0.5 \mathrm{wt} \%)$, PS layers with different thicknesses were prepared (spraying 1,3 , and 5 times, $30 \mu \mathrm{L}$ per time, the corresponding thicknesses were approximately 5, 15 and $25 \mu \mathrm{m}$, respectively, see Fig. 3b). As shown in Fig. 3a, the transmission intensities of the PS layers decreased as the spraying cycles increased. The transmittance was close to $0 \%$ in the entire visible region when the number of spraying cycles reached 5 . Hence, the thicknesses of PS layers were set to approximately $25 \mu \mathrm{m}$ for complete concealment of the middle layer in our designed composite structure. The optical shielding can be realized by adjusting the thickness of QASs for PS microspheres of other sizes. The transmittance of the 5 PS exhibited a little change before and after hot pressing (Fig. 3a). The corresponding transmission spectra of two TPU films showed that light transmittance was enhanced after hotpress molding, indicating that the two films became an integrated whole. The tight confinement protection of heterostructure prevents encrypted information from physical or chemical damage during transmission.

In the designed encryption mode (Fig. 3c), the observed color is related to the characteristic sizes of the shortrange-ordered PS layer, and the incident light cannot penetrate it due to its shielding effect. However, the increment in thickness enhances incoherent scattering from the interior of the quasi-amorphous arrays, making the coherent scattering of the characteristic wavelength not prominent [29,30]. The structural color exhibits low contrast and becomes nearly white (Fig. 3d). Therefore, we added a small amount of CB (0.5 wt $\%)$ as the absorption additive of background scattering to improve the saturation of the noniridescent structural color $[30,44]$. The optical shielding ability of the PS layer further improved with the addition of CB (Fig. 3e). As the particle sizes of PS microspheres increased, the intensity of Rayleigh scattering increased [36]. As a result of coherent scattering and Rayleigh scattering, the encoded composite structure presented a magenta color (see Fig. 3d).

The spherical morphology of PS microspheres is the key to realizing the optical shielding of QASs via multiple light scattering. If the morphology of PS microspheres changes, the light transmission will change [41]. Herein, light transmittance control by morphological change is utilized to achieve high-security decryption. PS microspheres were prepared by emulsion polymerization of styrene monomer. The linear polymer structure formed by double bond addition cannot play a rigid supporting role, and the $T_{\mathrm{g}}$ was approximately $95^{\circ} \mathrm{C}$ (measured by DSC, see Fig. $3 \mathrm{f}$ ). Therefore, the PS microsphere easily undergoes phase state transition and morphology change under high-temperature conditions. Under its $T_{\mathrm{g}}$, the polymer is in the glass state. Molecular chains cannot move, but the atoms (or groups) that constitute the molecules vibrate in their equilibrium positions [37]. However, the movement of chain segments induces elastic property at $T_{\mathrm{g}}$. When the temperature continues to increase, all of the molecular chains move and exhibit a 


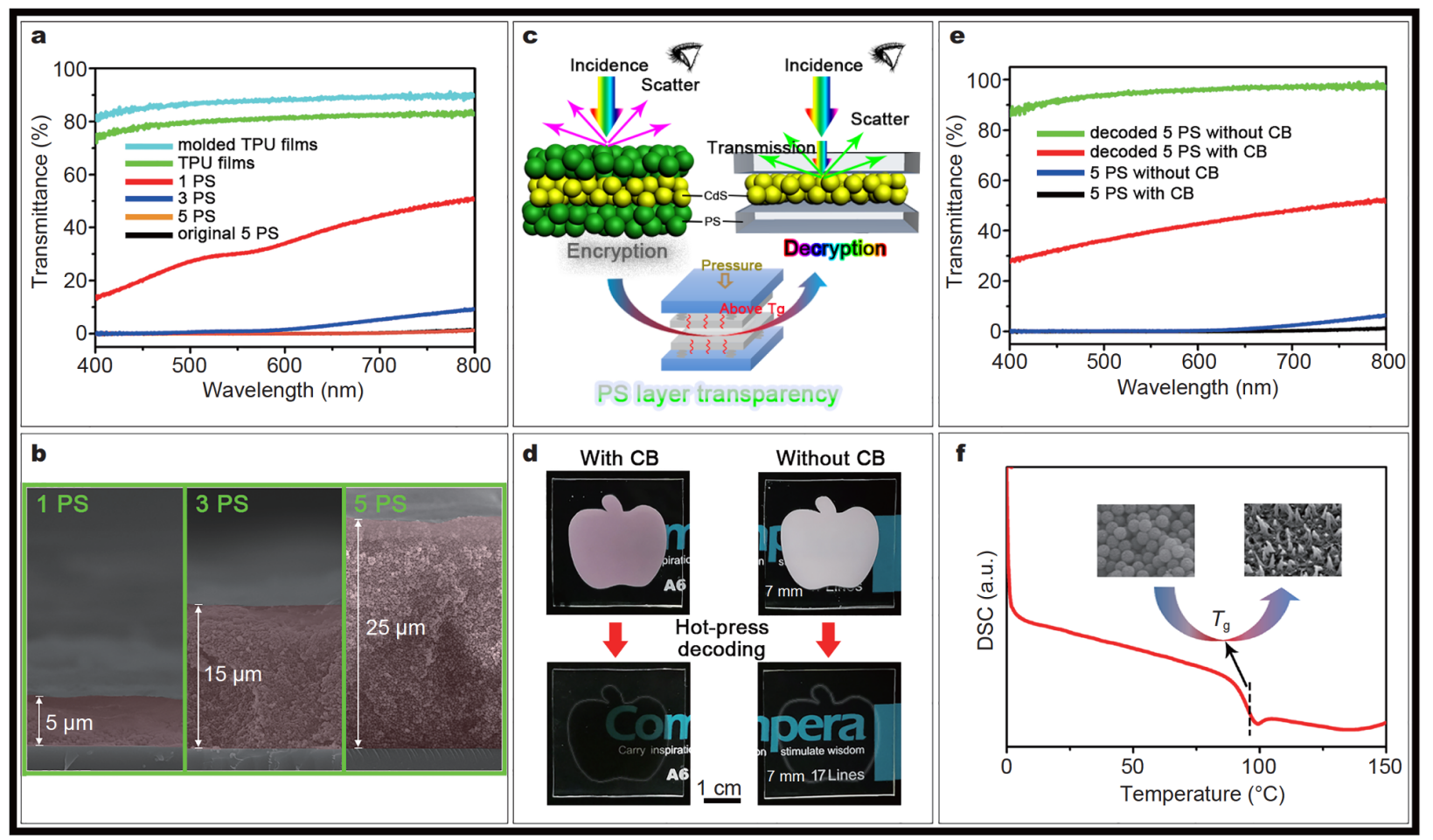

Figure 3 Mechanism of encryption and decryption for optical composite devices. (a) Transmission spectra of PS layers with different thicknesses and two TPU films before and after hot-press molding $\left(90^{\circ} \mathrm{C}\right.$ and $\left.8 \mathrm{kN}\right)$. "1-5 PS" means the PS layers formed by spraying 1-5 times. "Original 5 PS" means the PS layer formed by spraying 5 times before hot-press molding. (b) Cross-sectional SEM images of PS layers with different thicknesses. (c) The models of structural color generation in encryption and decryption modes. The decoding conditions are imperceptible in daily environment and thus difficult to crack during transmission, providing high-level information security. (d) Photos of the composite structure only containing 5 PS (with and without $0.5 \mathrm{wt} \% \mathrm{CB}$ ) before and after hot-press decoding $\left(120^{\circ} \mathrm{C}\right.$ and $\left.2 \mathrm{kN}\right)$. (e) Transmission spectra of PS layers (with and without $0.5 \mathrm{wt} \%$ $\mathrm{CB}$ ) before and after hot-press decoding $\left(120^{\circ} \mathrm{C}\right.$ and $\left.2 \mathrm{kN}\right)$. (f) DSC curve of PS microspheres. Inset is the SEM images of PS microspheres before and after phase state transition.

viscous flow property. In the molded composites, PS microspheres retained rigidity at $90^{\circ} \mathrm{C}$ (below $T_{\mathrm{g}}$ ) because of their glassy state, and their spherical shapes and the structural color of QASs maintained. When the final encoding structure was decrypted by hot pressing at high temperature (such as $120^{\circ} \mathrm{C}$, above the $T_{\mathrm{g}}$ of PS microspheres), the PS microspheres became increasingly deformed and turned into a viscous fluid (inset of Fig. 3f). As demonstrated in Fig. S3, decoding was rapid because the uniform and forced contact with the heating pad allowed fast heat transfer in the composite film under a certain pressure. Taking $2 \mathrm{kN}$ of hot pressing pressure as an example, the transparency transition of PS layers was realized within $15 \mathrm{~s}$ during decryption. In the original state at room temperature $(0 \mathrm{~s})$, the PS layers were magenta, completely covering the green CdS pattern.

When hot-pressed at $120^{\circ} \mathrm{C}$ and $2 \mathrm{kN}$, the encoded composite film became transparent, and the structural color of the middle flower pattern became clearly visible.
On the basis of the above experiments, the encrypted composite structure was placed into a preheated oven at $120^{\circ} \mathrm{C}$ in the absence of pressure. The PS layers were incompletely transparent, presenting a frosted look (Figs S4 and S5), and the hidden information cannot be recognized. According to its cross-sectional scanning electron microscopy (SEM) image (Fig. S6), the PS layers were heated slowly and unevenly without pressure, so the microspheres melted incompletely within $10 \mathrm{~min}$ and there were still some gaps among them. Based on the above results, we conclude that the temperature higher than $T_{\mathrm{g}}$ is the premise of triggering the morphology change of PS microspheres, while the pressure allows the composites to conduct heat uniformly in a short time to form a seamless and transparent PS layer.

Strategies of selectively exposing combination ciphers through deformation, solvent and angle change are prone to data breach during transmission or in a data storage medium. If decryption methods are easy to find in daily 
environment and thus crack, they are unsuitable for practical application in actual security devices. However, the single-time-use decoding under high-temperature conditions (the temperature above the $T_{\mathrm{g}}$ of PS microspheres and under pressure) is imperceptible (Fig. 3c), and provides high-level information security. To gain insight into the encryption security in the daily environment, we hot-pressed the encrypted samples at $60^{\circ} \mathrm{C}$. In these experiments, the sample hot-pressed under $2 \mathrm{kN}$ for $20 \mathrm{~min}$ did not exhibit the hidden CdS pattern. When the pressure was increased to $8 \mathrm{kN}$ (Fig. S7), the patterned region remained invisible over time. The system is also independent of angle and solvent, signifying its potential security function for encryption during transmission.

In the decryption mode, which is schematically shown in Fig. 3c, the revelation of the hidden pattern could be attributed to the transparency transition of PS layers under high-temperature (above the $T_{\mathrm{g}}$ of PS microspheres) conditions. The morphology change of PS microspheres eliminates the shielding effect of the multiple light scattering and allows the incident light and scattering light to penetrate them. The transmission spectra showed that the light transmittance of the PS layer was markedly enhanced after hot pressing at $120^{\circ} \mathrm{C}$ (Fig. 3e). The transmittance of the 5 PS without CB reached $90 \%$, and this behavior was consistent with the color change of the composite structure (Fig. 3d). Despite the introduction of $\mathrm{CB}$ for improving color saturation in the encryption mode, the shielding effect of the 5 PS with $\mathrm{CB}$ was largely reduced after decoding (Fig. 3e), which allowed the scattering light to penetrate the CdS layer and revealed the structural color pattern. Because of the high refractive index of 2.5, CdS microspheres were selected to assemble the encryption layer, and their average diameter is $182.1 \mathrm{~nm}$ (Fig. S2). The high refractive index caused coherent scattering to become prominent in the shortrange-ordered state $[39,40]$, so the structural color pattern of CdS microspheres was clearly observed in the decryption mode. Furthermore, the unique noniridescent structural colors of QASs composed of CdS microspheres provide unique spectral information, accounting for advanced information security application compared with ordinary pigments. The X-ray diffraction (XRD) patterns revealed the cubic phase of the CdS microspheres (Fig. S8). In this system (with CB), it is difficult to decipher the structure color of the microspheres with low refractive index (such as $\mathrm{SiO}_{2}$ ) in the short-range-ordered state. Other heat-resistant microspheres with high refractive index are also suitable for this encryption system, and the camouflage ability and imperceptible decryption method enable high-level security.

\section{Microstructures and optical properties}

The SEM images (Fig. 4a, c, Figs S9 and S10) show the morphology and microstructures after the layer-by-layer construction of trilayer QAS patterns. The SEM images present the closely packed composition of PS and CdS microspheres with a quasi-amorphous state. The corresponding scattering spectra (Fig. $4 \mathrm{~b}$ ) indirectly proved that PS layers realized the light shielding of the CdS layer. The boundaries between the different QAS layers can be observed in the cross-sectional SEM image (Fig. 4d). The trilayer structure was further confirmed by the corresponding energy-dispersive spectroscopy (EDS) mapping. The thicknesses of the upper and bottom PS layers were approximately $25 \mu \mathrm{m}$, whereas the thickness of the middle CdS layer was approximately $2 \mu \mathrm{m}$. As shown by the SEM image obtained under high magnification (Fig. 4g), the PS and CdS microspheres were arranged in a shortrange order, and the contact edges between the layers were distinct.

According to the procedures in Fig. 2, the final robust product with encrypted information was prepared under a hot pressing condition of $90^{\circ} \mathrm{C}$ and $8 \mathrm{kN}$ (Fig. 4e). Due to the delicate design of the trilayer structure and the multiple scattering effect of the PS layers [27-31], the final coded product showed a magenta apple pattern from its top and bottom views (Fig. $4 \mathrm{e}$ and Fig. S11). The single scattering peak observed in the scattering spectra (Fig. 4h) further showed that the encrypted patterns in our strategy were completely hidden because of the optical screening effect. Moreover, the noniridescence of the composite structure was indicated by the scattering spectra at different angles (Fig. S12). When the coded structure was hot-pressed at $120^{\circ} \mathrm{C}$, the PS layers covering the CdS pattern became transparent. As demonstrated by Fig. 4e, an obvious color change occurred, resulting in a sharp color contrast and hence the display of a green flower pattern. The scattering spectra (Fig. 4h) changed and showed another different scattering peak. The significant difference in the morphology of the PS layers, as illustrated by the comparison of corresponding profile SEM images and mapping (Fig. $4 \mathrm{~d}, \mathrm{f}, \mathrm{g}$ and $\mathrm{i}$ ), ensured the disappearance of multiple light scattering.

\section{Structural stability}

The conformational structure and molecular mobility of amorphous polymer surface are different from that of bulk, and the degree of molecular chain entanglement is 


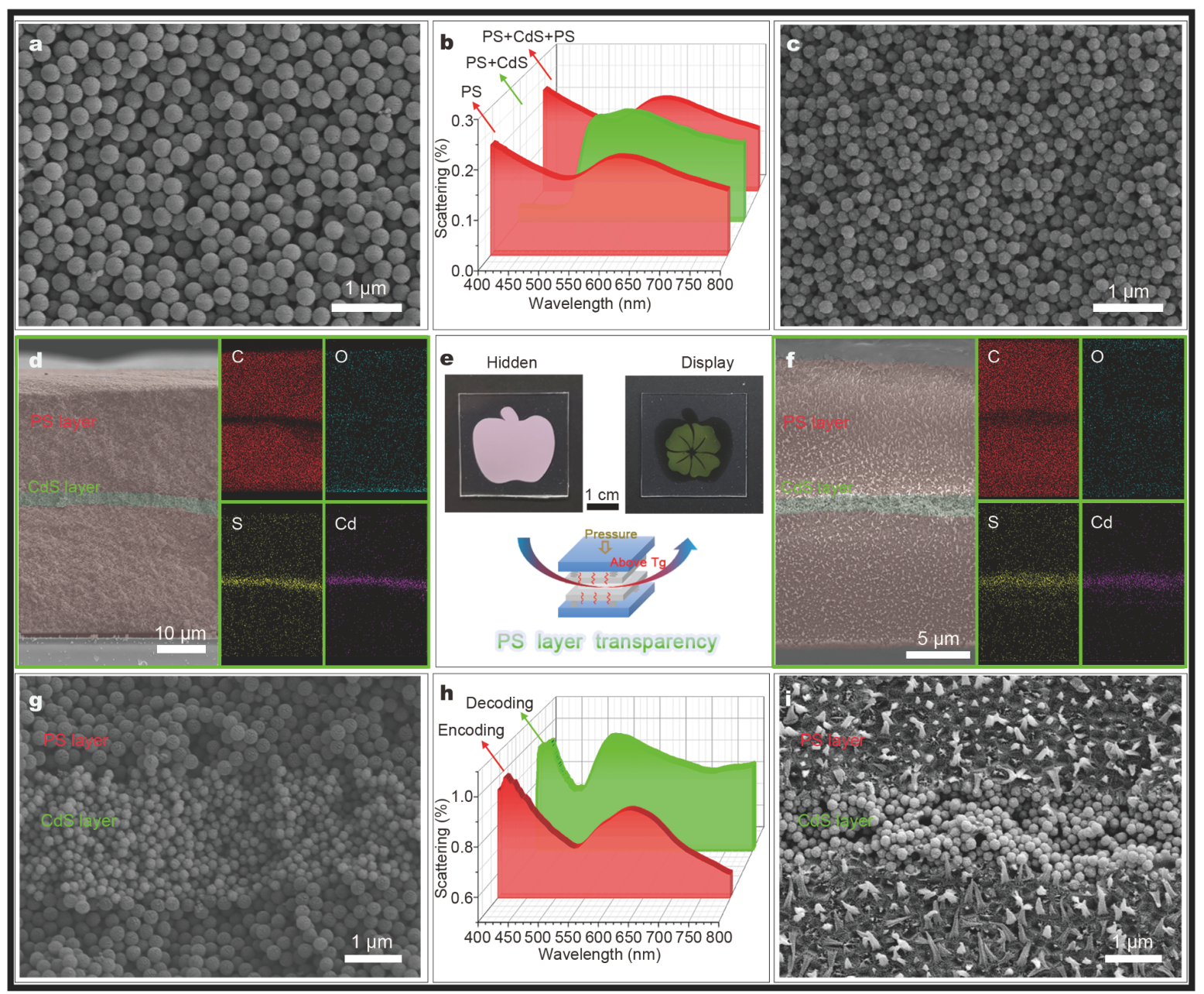

Figure 4 Microstructures and optical properties of optical composite devices. (a) Superficial SEM image of PS layer. (b) Scattering spectra of the multilayer structure (PS, PS+CdS, PS+CdS+PS). (c) Superficial SEM image of PS+CdS bilayer structure. (d) Cross-sectional SEM image of trilayer quasi-amorphous heterostructure and the corresponding elemental mapping. (e) Photos of the final encoded composite product before and after hotpress decoding $\left(120^{\circ} \mathrm{C}\right.$ and $2 \mathrm{kN}$ ). (f) Cross-sectional SEM image of the composite structure after hot-press decoding and the corresponding elemental mapping. (g) SEM image of the interfaces in (d) at high magnification. (h) Scattering spectra of the coding region in encoding and decoding modes (normalization). (i) SEM image of the interfaces in (f) at high magnification.

lower. Therefore, the chain of polymer surface layer is more fluid. When the temperature is higher than $T_{\mathrm{g}}$, a large number of macromolecular chain segments diffuse mutually at the interface of two films. The chain ends pass into the other side of the surface interface; thus adhesion occurs at the interface. In the process of hot-press molding, the diffusion of polymer chains at the interface of two TPU films leads to superadhesion, which realizes the encapsulation of QASs (Fig. S13). Furthermore, we investigated the durability of the final encryption composite product through a peeling test. Test strips prepared under different conditions were stretched at the rate of $50 \mathrm{~mm} \mathrm{~min}{ }^{-1}$ until completely peeled off as schematically shown in Fig. 5b. Under a fixed hot pressing pressure of $8 \mathrm{kN}$, the test strips prepared at 70 and $80^{\circ} \mathrm{C}$ can be easily peeled off completely (peeling force less than $100 \mathrm{~N}$, see Fig. 5a). When the hot pressing temperature exceeded $90^{\circ} \mathrm{C}$, peeling the test strips under the applied peeling force of $285 \mathrm{~N}$ became difficult. The mechanical strength of the samples prepared at a fixed hot pressing temperature of $90^{\circ} \mathrm{C}$ was also studied. Fig. $5 \mathrm{c}$ shows that the peeling force of the sample prepared under $4 \mathrm{kN}$ was about $175 \mathrm{~N}$, and stability further enhanced when the samples were prepared at $8 \mathrm{kN}$ and above. Comparison of the cross-sectional SEM images of the composite structures prepared under different hot pressing conditions 


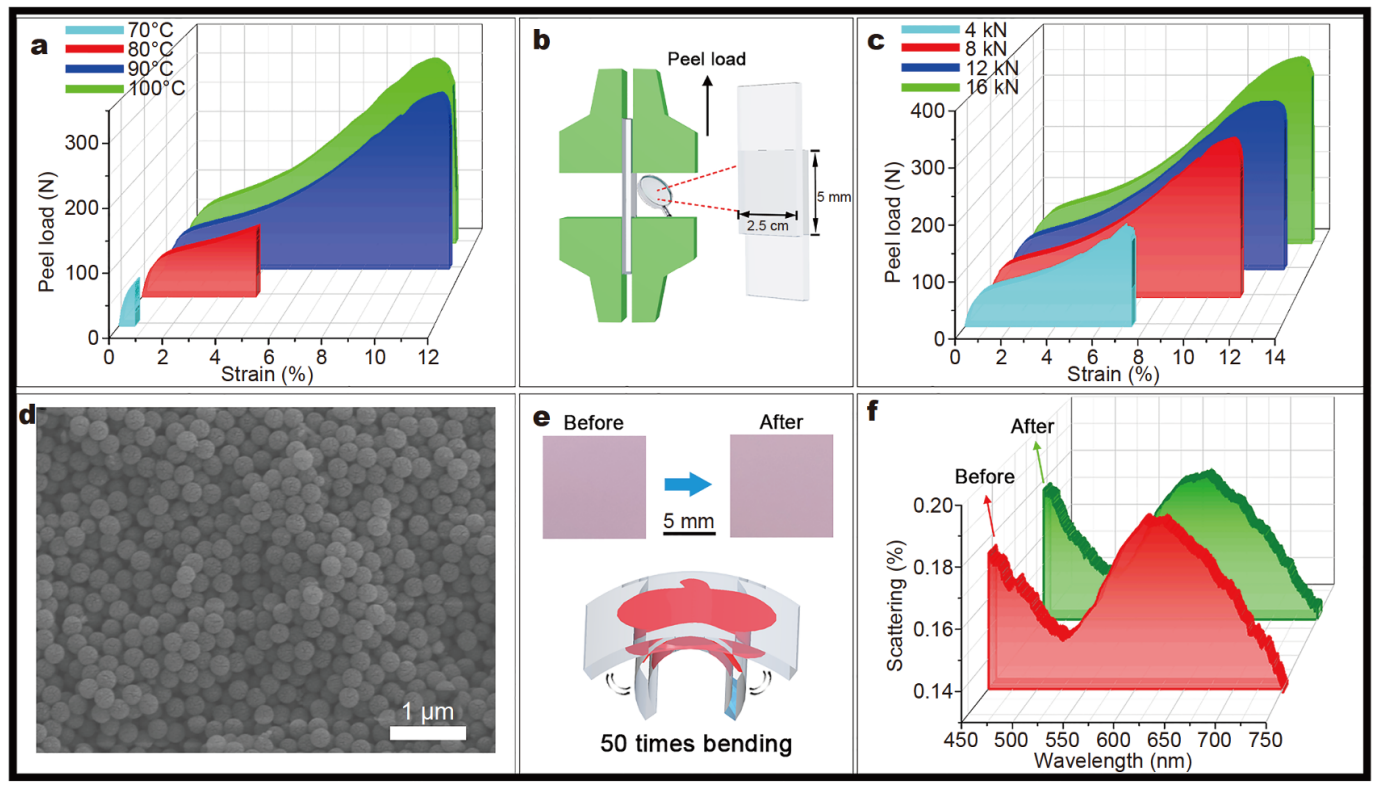

Figure 5 Performance of optical composite devices. (a) The peel load of the test strips prepared under various hot pressing conditions $\left(70-100^{\circ} \mathrm{C}\right.$, $8 \mathrm{kN}$ ). (b) Schematic diagram of peeling test. (c) The peel load of the test strips prepared under various hot pressing conditions $\left(90^{\circ} \mathrm{C}, 4-16 \mathrm{kN}\right)$. (d) Cross-sectional SEM image of PS layer after hot-press molding $\left(90^{\circ} \mathrm{C}, 8 \mathrm{kN}\right)$. (e) Photos of the composite structure before and after the bending test. Inset is the schematic diagram of bending test. (f) The corresponding scattering spectra before and after 50 times bending.

revealed that PS microspheres maintained good spherical morphology when hot-pressed below $90^{\circ} \mathrm{C}$ (Fig. $5 \mathrm{~d}$ and Fig. S14). However, the microspheres began to deform at $100^{\circ} \mathrm{C}$, and inorganic $\mathrm{CdS}$ microspheres were still stable at $120^{\circ} \mathrm{C}$ (Fig. $4 \mathrm{i}$ ). Therefore, in our designed structure, the hot pressing condition of $90^{\circ} \mathrm{C}$ and $8 \mathrm{kN}$ was selected for the preparation of the encoded composite products with robust mechanical stability. The bending experiments (Fig. 5e, f) demonstrate the stability and flexibility of the encryption product under this molding condition. Almost no difference in scattering curves was observed after the sample was bent 50 times $(5 \mathrm{~mm}$ bending radian). The excellent mechanical properties of our designed encryption product confirmed its suitability for true and persistent information hiding for security purposes.

\section{Encryption application}

The encrypted layer can be further patterned through the precise design of complex combinatorial codes with identification shapes. The quick response $(\mathrm{QR})$ code is a graphic that records the information of data symbols. Specific geometric figures are distributed in plane (twodimensional direction) in accordance with certain laws [45]. A number of geometric forms corresponding to binary codes are used to represent the numerical in- formation of character; this information can be read automatically by an image input device or a photoelectric scanning device for automatic information processing. It has the advantages of high-density coding, large information capacity, wide coding range, and high decoding reliability, and these features are especially suitable for security and information tracking [45,46]. A QR code layer of CdS was introduced into the composite structure through the spraying method, and the final encryption product was prepared through hot pressing (Fig. 6a). Notably, the inside pattern was completely hidden under normal conditions. The graphic that retained the details was displayed after hot-press decoding. This behavior is a promising feature for strict security protection. The visual encryption and decryption based on spraying can be used for information recognition. The decrypted QR code was successfully scanned and read out with a smartphone (Video S1), presenting the implied information (Dalian University of Technology). Interestingly, the trilayer quasi-amorphous heterostructure with invisible and complex encrypted information was easily compounded into a TPU phone case by hot-press molding (Fig. 6b), indicating that the strategy can be applied to some portable information carriers with excellent durability and flexibility (demonstrated by the bending test in Fig. S15). Therefore, the capability to integrate with daily life ma- 
terials provides strong camouflage for portable composites, which can further enhance information security.

\section{CONCLUSIONS}

In summary, a trilayer quasi-amorphous heterostructure for the encryption and decryption of complex information based on optical shielding properties and phase state transition is reported for the first time. This heterostructure can be composited in TPU films for the preparation of portable and camouflaged products. In the encoding mode, the multiple light scattering of PS microspheres can effectively block the transmission of light, and nearly $0 \%$ transmittance can be achieved by controlling the thicknesses of the upper and bottom PS layers. Therefore, the composite structure only displayed the structural color of the PS layer, and the middle CdS encrypted pattern was completely hidden without color contrast. Decoding under high-temperature conditions transformed the PS layers into a transparent state. This phenomenon allowed the coherent scattering of $\mathrm{CdS}$ microspheres with high refractive index to pass through and revealed the structural color patterns in detail. Importantly, the decryption method is imperceptible in daily environment, avoiding data breach during transmission and providing high-level information security. The mol-

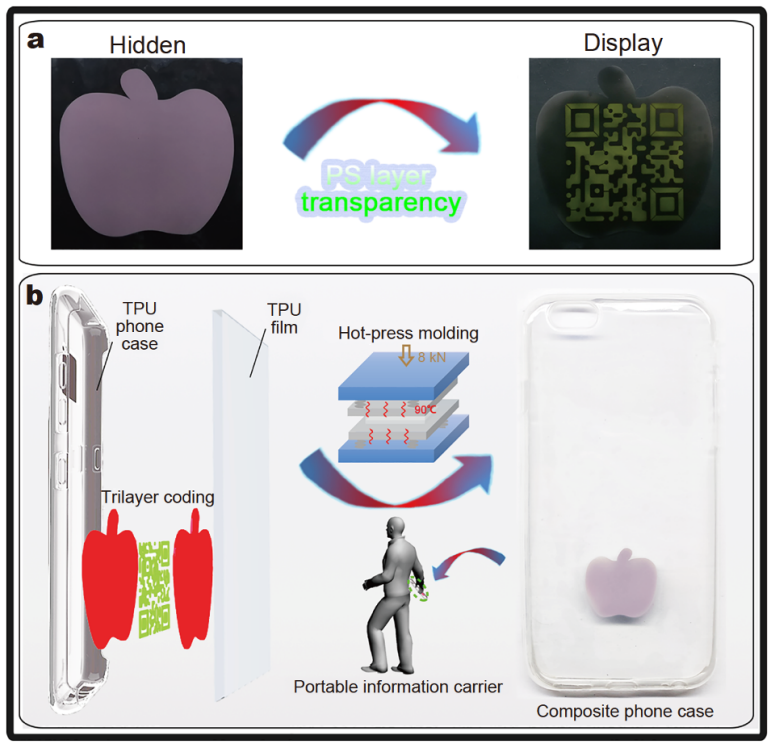

Figure 6 Visual encryption and camouflage of optical composite devices. (a) Photos of the encryption composite structure with QR code pattern of CdS in encryption and decryption modes. (b) Schematic diagram to fabricate TPU composite phone case with trilayer patterned quasi-amorphous heterostructure: trilayer QAS patterns were constructed on a TPU film, and then the composite structure of TPU film and TPU phone case was treated in a hot-press apparatus $\left(90^{\circ} \mathrm{C}, 8 \mathrm{kN}\right)$. ded thermoplastic composites kept good mechanical stability after the peeling and bending tests. Moreover, complex information can be easily loaded into a composite phone case for convenient camouflage. The high encoding and decoding security, excellent mechanical performance and flexibility, and easy loading into portable carriers indicate great application value in the field of information security, such as encryption, anti-counterfeiting and cryptography.

\section{Received 28 May 2020; accepted 24 August 2020;}

published online 10 November 2020

1 Song Z, Lin T, Lin L, et al. Invisible security ink based on watersoluble graphitic carbon nitride quantum dots. Angew Chem Int Ed, 2016, 55: 2773-2777

2 Hou X, Ke C, Bruns CJ, et al. Tunable solid-state fluorescent materials for supramolecular encryption. Nat Commun, 2015, 6: 6884

3 Liu J, Wang N, Yu Y, et al. Carbon dots in zeolites: A new class of thermally activated delayed fluorescence materials with ultralong lifetimes. Sci Adv, 2017, 3: e1603171

4 Xu S, Chen R, Zheng C, et al. Excited state modulation for organic afterglow: Materials and applications. Adv Mater, 2016, 8: 99209940

5 Jiang K, Zhang L, Lu J, et al. Triple-mode emission of carbon dots: Applications for advanced anti-counterfeiting. Angew Chem Int Ed, 2016, 55: 7231-7235

6 Li W, Wang Y, Li M, et al. Inkjet printing of patterned, multispectral, and biocompatible photonic crystals. Adv Mater, 2019, 31: 1901036

7 Qi Y, Niu W, Zhang S, et al. Encoding and decoding of invisible complex information in a dual-response bilayer photonic crystal with tunable wettability. Adv Funct Mater, 2019, 29: 1906799

8 Zhong K, Li J, Liu L, et al. Instantaneous, simple, and reversible revealing of invisible patterns encrypted in robust hollow sphere colloidal photonic crystals. Adv Mater, 2018, 30: 1707246

9 Peng CY, Hsu CW, Li CW, et al. Flexible photonic crystal material for multiple anticounterfeiting applications. ACS Appl Mater Interfaces, 2018, 10: 9858-9864

10 Heo Y, Kang H, Lee JS, et al. Lithographically encrypted inverse opals for anti-counterfeiting applications. Small, 2016, 12: 38193826

$11 \mathrm{Hu} \mathrm{H}$, Zhong $\mathrm{H}$, Chen $\mathrm{C}$, et al. Magnetically responsive photonic watermarks on banknotes. J Mater Chem C, 2014, 2: 3695-3702

12 He X, Gu Y, Yu B, et al. Multi-mode structural-color anti-counterfeiting labels based on physically unclonable amorphous photonic structures with convenient artificial intelligence authentication. J Mater Chem C, 2019, 7: 14069-14074

13 Xuan R, Ge J. Invisible photonic prints shown by water. J Mater Chem, 2012, 22: 367-372

14 Chen K, Zhang Y, Ge J. Highly invisible photonic crystal patterns encrypted in an inverse opaline macroporous polyurethane film for anti-counterfeiting applications. ACS Appl Mater Interfaces, 2019, 11: 45256-45264

15 Yang B, Li L, Du K, et al. Photo-responsive photonic crystals for broad wavelength shifts. Chem Commun, 2018, 54: 3057-3060

16 Shang L, Zhang W, Xu K, et al. Bio-inspired intelligent structural 
color materials. Mater Horiz, 2019, 6: 945-958

17 Ge D, Lee E, Yang L, et al. A robust smart window: Reversibly switching from high transparency to angle-independent structural color display. Adv Mater, 2015, 27: 2489-2495

18 Fu F, Chen Z, Wang H, et al. Graphene hybrid colloidal crystal arrays with photo-controllable structural colors. Nanoscale, 2019, 11: 10846-10851

19 Meng ZJ, Zhang J, Deng X, et al. Bioinspired hydrogel microfibres colour-encoded with colloidal crystals. Mater Horiz, 2019, 6: 19381943

20 Gu M, Zhang Q, Lamon S. Nanomaterials for optical data storage. Nat Rev Mater, 2016, 1: 16070

21 Du J, Sheng L, Chen Q, et al. Simple and general platform for highly adjustable thermochromic fluorescent materials and multifeasible applications. Mater Horiz, 2019, 6: 1654-1662

22 Liu X, Wang J, Tang L, et al. Flexible plasmonic metasurfaces with user-designed patterns for molecular sensing and cryptography. Adv Funct Mater, 2016, 26: 5515-5523

23 Zijlstra P, Chon JWM, Gu M. Five-dimensional optical recording mediated by surface plasmons in gold nanorods. Nature, 2009, 459: $410-413$

24 Duan X, Kamin S, Liu N. Dynamic plasmonic colour display. Nat Commun, 2017, 8: 14606

25 Qi Y, Chu L, Niu W, et al. New encryption strategy of photonic crystals with bilayer inverse heterostructure guided from transparency response. Adv Funct Mater, 2019, 29: 1903743

26 Meng Y, Qiu J, Wu S, et al. Biomimetic structural color films with a bilayer inverse heterostructure for anticounterfeiting applications. ACS Appl Mater Interfaces, 2018, 10: 38459-38465

27 Yoshioka S, Takeoka Y. Production of colourful pigments consisting of amorphous arrays of silica particles. ChemPhysChem, 2014, 15: 2209-2215

28 Wu S, Liu T, Tang B, et al. Different structural colors or patterns on the front and back sides of a multilayer photonic structure. ACS Appl Mater Interfaces, 2019, 11: 27210-27215

29 Iwata M, Teshima M, Seki T, et al. Bio-inspired bright structurally colored colloidal amorphous array enhanced by controlling thickness and black background. Adv Mater, 2017, 29: 1605050

30 Takeoka Y, Yoshioka S, Takano A, et al. Production of colored pigments with amorphous arrays of black and white colloidal particles. Angew Chem Int Ed, 2013, 52: 7261-7265

31 Wu S, Liu T, Tang B, et al. Structural color circulation in a bilayer photonic crystal by increasing the incident angle. ACS Appl Mater Interfaces, 2019, 11: 10171-10177

32 Zhang J, Meng Z, Liu J, et al. Spherical colloidal photonic crystals with selected lattice plane exposure and enhanced color saturation for dynamic optical displays. ACS Appl Mater Interfaces, 2019, 11: 42629-42634

33 Kim SH, Park JG, Choi TM, et al. Osmotic-pressure-controlled concentration of colloidal particles in thin-shelled capsules. Nat Commun, 2014, 5: 3068

34 Noh H, Liew SF, Saranathan V, et al. Double scattering of light from biophotonic nanostructures with short-range order. Opt Express, 2010, 18: 11942-11948

35 Li Q, Zhang Y, Shi L, et al. Additive mixing and conformal coating of noniridescent structural colors with robust mechanical properties fabricated by atomization deposition. ACS Nano, 2018, 12: 3095-3102

36 Ge D, Yang L, Wu G, et al. Angle-independent colours from spray coated quasi-amorphous arrays of nanoparticles: combination of constructive interference and rayleigh scattering. J Mater Chem C, 2014, 2: 4395-4400

37 Dechun Z. 4-Chemical and photophysical properties of materials for OLEDs. In: Buckley A (eds.). Org Light-Emit Diodes OLEDs. Cambridge: Woodhead Publishing, 2013, 114-142

38 Wu X, Hong R, Meng J, et al. Hydrophobic poly(tert-butyl acrylate) photonic crystals towards robust energy-saving performance. Angew Chem, 2019, 131: 13690-13698

39 Wu S, Liu B, Su X, et al. Structural color patterns on paper fabricated by inkjet printer and their application in anticounterfeiting. J Phys Chem Lett, 2017, 8: 2835-2841

40 Braun PV, Wiltzius P. Electrochemically grown photonic crystals. Nature, 1999, 402: 603-604

41 Meng F, Umair MM, Zhang S, et al. Thermal-guided interfacial confinement to fabricate flexible structural color composites for durable applications. J Mater Chem C, 2019, 7: 11258-11264

42 Huang Y, Zhou J, Su B, et al. Colloidal photonic crystals with narrow stopbands assembled from low-adhesive superhydrophobic substrates. J Am Chem Soc, 2012, 134: 17053-17058

43 Zhou J, Han P, Liu M, et al. Self-healable organogel nanocomposite with angle-independent structural colors. Angew Chem Int Ed, 2017, 56: 10462-10466

44 Zhang Y, Han P, Zhou H, et al. Highly brilliant noniridescent structural colors enabled by graphene nanosheets containing graphene quantum dots. Adv Funct Mater, 2018, 28: 1802585

45 Barrera JF, Mira A, Torroba R. Optical encryption and QR codes: Secure and noise-free information retrieval. Opt Express, 2013, 21: 5373-5378

46 Heydari E, Sperling JR, Neale SL, et al. Plasmonic color filters as dual-state nanopixels for high-density microimage encoding. Adv Funct Mater, 2017, 27: 1701866

Acknowledgements This work was financially supported by the National Natural Science Foundation of China (21878043, 21576039, 21421005 and U1608223), the Program for Innovative Research Team in University (IRT_13R06), the Fundamental Research Funds for the Central Universities (DUT18ZD218), and the Talent Fund of Shandong Collaborative Innovation Center of Eco-Chemical Engineering (XTCXYX04).

Author contributions Meng F and Tang B designed and engineered the samples; Meng F and Malik MM performed the experiments; Meng F wrote the paper with support from Zhang S, Meng Y, and Tang B. All authors contributed to the general discussion.

Conflict of interest The authors declare that they have no conflict of interest.

Supplementary information Experimental details and supporting data are available in the online version of the paper. 


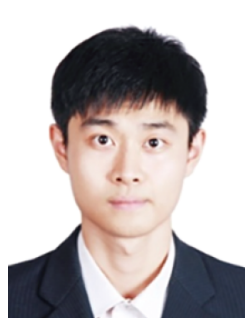

Fantao Meng is currently a master student under the supervision of Prof. Bingtao Tang at Dalian University of Technology. His current research interests focus on the micro/nano interface and structural color.

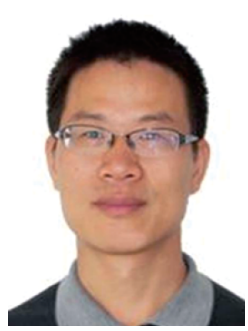

Bingtao Tang is currently a Professor at Dalian University of Technology. He received his Doctorate from Dalian University of Technology in 2005, and became a Professor in 2016. His research interests include energy conversion and storage, micro/nano interface and structural color, dyestuff chemistry and photochemistry. During his career, his research has resulted in 100 papers and 18 patents.

\section{简便制备具有三层准晶异质结构的加密复合材料}

孟繁涛, Malik Muhammad Umair ${ }^{1}$, 张淑芬 ${ }^{1}$, 孟尧 ${ }^{2}$, 唐炳涛 ${ }^{1,2 *}$ 摘要 加密材料是信息安全领域的一个主要研究方向, 然而将复杂 编码的完全隐藏、传输过程中的稳定性、伪装性和可靠的解码方 法相结合来实现理想的信息安全, 仍具有挑战性. 本文报道了一种 创新策略, 用于合理构建具有高安全性和便携性的加密复合材料. 与普通的胶体光子水印相比, 这种加密光学器件由热塑性聚氨酯 (TPU)保护层、聚苯乙烯( PS)转换层和 CdS 编码层组成. 通过热激 活的透光率控制, PS层的光屏蔽作用和相态转变使复合材料具有 高级别的编解码安全性. 同时, 由于高折射率, CdS层在解码模式下 可以清晰地显示出来. 此外, TPU膜的限域保护能够有效防止三层 准晶异质结构的破损. 为了建立实际应用优势, 可以制备便携式信 息载体进行伪装. 因此, 可靠的编解码体系以及与便携性载体相结 合的能力, 使这种高安全性加密器件具有应用于反欺计领域的潜 力. 